\title{
Normal Immunostaining Pattern for Aquaporin-5 in the Lesions of Palmoplantar Hyperhidrosis
}

\author{
Kyoko Nakahigashi Takashi Nomura Yoshiki Miyachi \\ Kenji Kabashima \\ Department of Dermatology, Graduate School of Medicine, Kyoto University, Kyoto, Japan
}

\section{Key Words}

Palmoplantar hyperhidrosis · Aquaporin-5

\begin{abstract}
Aquaporin-5 (AQP-5) is a water-transporting protein expressed in mammal sweat glands. It has been reported that the expression of AQP-5 is involved in sweating of mice, rats, and horses. However, the physiological function of human AQP-5 is still uncertain. In this report, we examined the expression pattern of AQP-5 in the skin lesions of palmoplantar hyperhidrosis in patients with Nagashima-type palmoplantar hyperkeratosis (PPK). We found that there was no significant difference in AQP-5 expression in the palmoplantar skin of healthy subjects and patients with palmoplantar hyperhidrosis. Our findings suggest that a mechanism other than AQP-5 may be involved in the pathogenesis of hyperhidrosis in PPK.
\end{abstract}

\section{Introduction}

Aquaporin $(\mathrm{AQP})$ is a water-transporting protein that has a variety of physiological functions in the skin [1-3]. Among AQPs, AQP-5 is known to be expressed in mammalian sweat glands. It has been reported that the expression of AQP-5 is involved in the sweating of mice, rats, and horses [4]. The physiological function of human AQP-5, however, remains unknown. In humans, AQP-5 is expressed in the sweat glands. While AQP-5 was reported to be aberrant in the sweat glands in aquagenic wrinkling of the palms [5], others reported that its expression was not affected in the lesions of idiopathic segmental anhidrotic scalp skin and primary hyperhidrotic axillary skin $[4,6]$. Therefore, it remains unclear whether the AQP-5 expression profile is related to the abnormal regulation of the sweat glands.

Kenji Kabashima, MD, PhD

Department of Dermatology

Graduate School of Medicine, Kyoto University

54 Kawahara-cho, Shogoin, Sakyo-ku, Kyoto 606-8507 (Japan)

E-Mail kaba@kuhp.kyoto-u.ac.jp 
Meanwhile, it is known that some kinds of palmoplantar hyperkeratosis (PPK), especially Unna-Thost-type and Nagashima-type, accompany palmoplantar hyperhidrosis [7, 8].

Herein, we evaluated human AQP-5 expression in the lesions of palmoplantar hyperhidrosis in PPK.

\section{Case Presentations}

During a 3-year period (2006-2008), a diagnosis of palmoplantar hyperhidrosis with PPK was made in 7 patients ( 4 males and 3 females, mean age \pm standard deviation $34.6 \pm$ 22.8 years, range 8-67). Skin samples were taken from the palms and soles of those patients and the healthy subjects ( 2 males and 4 females, mean age $51.5 \pm 15.9$ years, range 27-66). Deparaffinized sections were immersed in $10 \mathrm{mM}$ sodium citrate solution for $10 \mathrm{~min}$ at $96^{\circ} \mathrm{C}$ for antigen retrieval. Endogenous peroxidase activity was blocked by $0.3 \%$ hydrogen peroxide for $30 \mathrm{~min}$. Staining for AQP-5 was incubated with rabbit polyclonal anti-AQP-5 antibody (AB3559; Millipore, Billerica, Mass., USA) at a dilution of 1:200 for $1 \mathrm{~h}$ at room temperature. Slides were incubated with biotinylated anti-rabbit IgG, and then with horseradish peroxidase-conjugated $\mathrm{ABC}$ reagent (Vector Laboratories Inc., Burlingame, Calif., USA). Coloring reaction was performed with a conventional substrate for diaminobenzidine (Vector Laboratories Inc.). This study was carried out in accordance with the Declaration of Helsinki, and was approved by the ethics committee of the Kyoto University. Informed consent was obtained from all patients enrolled in the study. The results showed that AQP-5 was expressed in the plasma membrane of the clear cells in eccrine sweat glands and the luminal plasmalemma of the dark cells. There was no significant histological difference between palmoplantar hyperhidrosis and healthy subjects (fig. 1).

\section{Discussion}

It has been reported that $\mathrm{AQP}-5$ protein is present in the apical membrane and cytoplasm of the dark cells in the secretory sweat coil [5], and that it is also expressed in the plasma membrane of clear cells $[4,6]$. The aberrant expression of AQPs may be derived from the translocation from intracellular vesicles to the plasma membrane by external stimuli to regulate the membrane permeability [9].

In this report, there was no significant difference in AQP-5 expression in the palmoplantar skin of healthy subjects and patients with palmoplantar hyperhidrosis. In addition, a recent study reported that there was no linkage to AQP-5 gene loci and primary focal hyperhidrosis, which is a disorder involving excessive sweating [10]. Therefore, a mechanism other than AQP-5 may be involved in the pathogenesis of hyperhidrosis in PPK. Further analysis on the expression pattern of AQP-5 in other hyper- or hypohidrosis patients is warranted.

\section{Acknowledgement}

This work was supported in part by Grants-in-Aid for Scientific Research from the Ministries of Education, Culture, Sports, Science and Technology and Health, Labor, and Welfare of Japan. 
Nakahigashi et al:: Normal Immunostaining Pattern for Aquaporin-5 in the Lesions of Palmoplantar Hyperhidrosis

\section{Disclosure Statement}

The authors have no conflicts of interest to declare.

\section{References}

1 Hara-Chikuma M, Chikuma S, Sugiyama Y, Kabashima K, Verkman AS, Inoue S, Miyachi Y: Chemokinedependent $\mathrm{T}$ cell migration requires aquaporin-3-mediated hydrogen peroxide uptake. J Exp Med 2012;209:1743-1752.

-2 Hara-Chikuma M, Sugiyama Y, Kabashima K, Sohara E, Uchida S, Sasaki S, Inoue S, Miyachi Y: Involvement of aquaporin-7 in the cutaneous primary immune response through modulation of antigen uptake and migration in dendritic cells. FASEB J 2012;26:211-218.

3 Nakahigashi K, Kabashima K, Ikoma, Verkman AS, Miyachi Y, Hara-Chikuma M: Upregulation of aquaporin-3 is involved in keratinocyte proliferation and epidermal hyperplasia. J Invest Dermatol 2011;131:865-873.

4 Iizuka T, Suzuki T, Nakano K, Sueki H: Immunolocalization of aquaporin-5 in normal human skin and hypohidrotic skin diseases. J Dermatol 2012;39:344-349.

5 Kabashima K, Shimauchi T, Kobayashi M, Fukamachi S, Kawakami C, Ogata M, Kabashima R, Mori T, Ota T, Fukushima S, Hara-Chikuma M, Tokura Y: Aberrant aquaporin 5 expression in the sweat gland in aquagenic wrinkling of the palms. J Am Acad Dermatol 2008;59:S28-S32.

-6 Bovell DL, MacDonald A, Meyer BA, Corbett AD, MacLaren AM, Holmes SL, Harker M: The secretory clear cell of the eccrine sweat gland as the probable source of excess sweat production in hyperhidrosis. Exp Dermatol 2011;20:1017-1020.

7 Lucker GP, Van de Kerkhof PC, Steijlen PM: The hereditary palmoplantar keratoses: an updated review and classification. Br J Dermatol 1994;131:1-14.

$>8$ Kabashima K, Sakabe J, Yamada Y, Tokura Y: 'Nagashima-type' keratosis as a novel entity in the palmoplantar keratoderma category. Arch Dermatol 2008;144:375-379.

$\checkmark 9$ Conner AC, Bill RM, Conner MT: An emerging consensus on aquaporin translocation as a regulatory mechanism. Mol Membr Biol 2013;30:1-12.

10 Del Sorbo F, Brancati F, De Joanna, Valenter EM, Lauria G, Albanese A: Primary focal hyperhidrosis in a new family not linked to known loci. Dermatology 2011;223:335-342.

isotype control

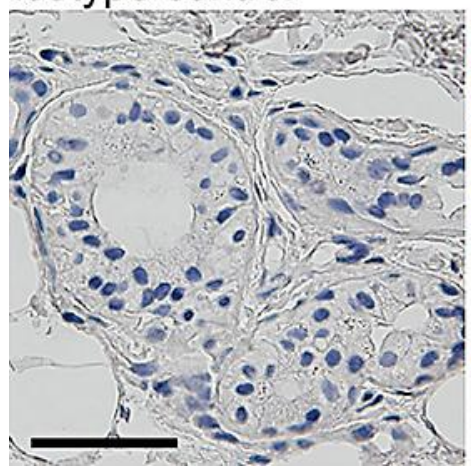

healthy subject

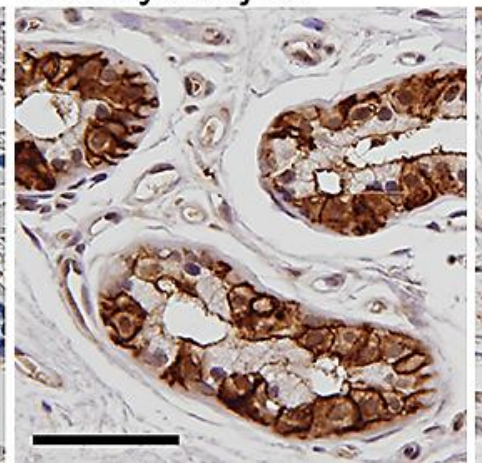

palmar hyperhidrosis

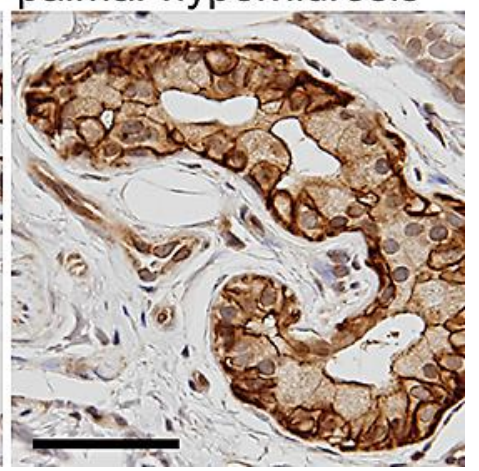

Fig. 1. Representative images of immunostaining for AQP-5 in human eccrine sweat glands taken from the palms of healthy subjects and patients with palmoplantar hyperhidrosis. Scale bar $=50 \mu \mathrm{m}$. 